\title{
DEVELOPMENT OF A MAGNETIC FLEXIBLE MEMBRANE MICROPUMP
}

\author{
Melvin Khoo, Liang-Hsuan Lu, and Chang Liu \\ Department of Electrical and Computer Engineering, University of Illinois at Urbana-Champaign \\ Urbana, IL 61801-2355
}

\section{SUMMARY}

We present results on the development of a micromachined magnetic membrane pump applicable to integrated microfluidic systems. Structurally, the pump consists of a magnetic microactuator and two polymer-based, one-way diffuser valves. The microactuator is based on a $40-\mu \mathrm{m}$ thick, flexible membrane made of poly dimethyl siloxane (PDMS), a soft silicone elastomer. Membrane displacement is caused by the interaction between an external magnet and ferromagnetic (Permalloy) pieces, embedded within the thickness of the membrane. Pump operation is simple as no precise magnet-to-pump alignment is required. Under an oscillating $1.99 \times 10^{5}-\mathrm{A} / \mathrm{m}$ external magnetic field, a $0.26-\mu 1 / \mathrm{min}$ flow rate was measured at an actuation frequency of $3.72-\mathrm{Hz}$.

The core component of the micropump is the magnetic microactuator. We have developed a novel micromachining process for embedding Permalloy pieces within a flexible, suspended PDMS membrane. The dimensions and placement of these Permalloy pieces within the membrane were optimized using computer simulations. Under a $1.84 \times 10^{5}-\mathrm{A} / \mathrm{m}$ magnetic field, the $2 \times 2-\mathrm{mm}^{2}$ PDMS membrane achieved a maximum vertical displacement of $211.5-\mu \mathrm{m}$.

\section{INTRODUCTION}

Micro liquid handling systems [1], in which pumps and valves are key components, have seen great interest during recent years. Actuation principles employed include thermopneumatic, piezoelectric, electrostatic, and electromagnetic. To achieve desirable large membrane displacements, relatively high voltages and high power are needed for these devices. Most membranes use silicon or silicon-based thin films, which have high Young's modulus and are therefore stiff. The maximum displacements of membrane actuators used in pumps and valves are generally limited (tens of microns), whether for flat membranes or even for specially designed membranes with corrugations. This property limits the overall volume flow rate of membrane micropumps.

A new magnetic membrane actuator has been developed. In comparison, the actuator achieves significantly larger membrane displacements than that of typical membrane actuators. This significantly larger displacement is affected by the use of PDMS, which has a Young's modulus 5 orders of magnitude lower than that of silicon. Besides this, PDMS is physically stable and chemically inert, making it more bio-compatible than silicon for biomedical applications. In addition, magnetic actuation is favorable as it provides large and long-range forces.

\section{PRINCIPLE AND DESIGN OF THE PUMP}

The micropump consists of two bonded layers (Fig. 1). The microfluidic circuit, Layer I, includes two one-way-flow diffuser valves connected in series with a pump chamber, which is linked by microchannels to the fluid inlet and outlet. The actuation unit, Layer II, is a magnetic membrane microactuator. The diffuser valves in Layer I maximize one-way fluid flow into and out of the pump chamber, in the direction denoted by the arrows. When a magnetic field is applied, the membrane deflects as shown in Figure 1. This pushes fluid out of the pump chamber through the outlet diffuser. When the external magnetic field is removed, the membrane relaxes and returns to its flat, at-rest position. This draws fluid in through the inlet diffuser to refill the pump chamber.

(A) Membrane Actuator. The structure of the actuator is shown in Figure 2. A thin layer of PDMS rests on top of a silicon cavity. Permalloy pieces, arranged in a parallel order on one side of the membrane, are embedded within the membrane. Interaction between an externally applied magnetic field with the Permalloy pieces causes membrane displacement. The applied field generates magnetic torque in the Permalloy and causes the pieces to deflect, thereby displacing the membrane as shown in Figure 1. Hence, a net volume displacement, or the stroke volume, is produced.

A highly desirable pump characteristic is to achieve the largest possible stroke volume, with a given magnetic field and membrane dimensions. Several unique design issues have been identified and optimized. These key design parameters include: (1) the size of Permalloy pieces, (2) the number of pieces, (3) the size of the membrane, and (4) the placement of Permalloy pieces within the membrane. Finite element analysis optimization simulations using ANSYS yielded the best design layout shown in Fig. 3(a).

(B) Diffuser Valve. The design of our diffuser valves follow the simulation and measurement studies by Olsson et. al. [2]. These no-moving-parts diffuser valves have diverging walls in the positive flow direction (Fig. 3b). Diffuser valves used in this pump are shown in Figure 3(b).

\section{FABRICATION}

(A) Membrane Actuator. Major steps in the fabrication process are illustrated in Figure 4. Etch holes are opened in the oxide of a silicon wafer. Bulk silicon etching is performed in EDP anisotropic wet silicon etchant until a thin, 30- $\mu \mathrm{m}$ layer of silicon is left (Fig. 4a). A 100- $\AA \mathrm{Cr}$ adhesion layer and a 2000- $\AA \mathrm{Cu}$ seed layer is then evaporated onto the front surface (Fig. $4 \mathrm{~b}$ ).

A $10-\mu \mathrm{m}$ thick layer of AZ4620 photoresist is spin-coated on the metals and then patterned for electroplating. Permalloy $\left(\mathrm{Ni}_{80} \mathrm{Fe}_{20}\right)$ is electroplated over the PR mold to a total height of 22$\mu \mathrm{m}$ (Fig. $4 \mathrm{~b}$ ). This intentional over-electroplating causes the plated Permalloy to extend laterally over the PR mold edges. The shape of the over-plated structure is shown in Fig. 5. The extension later provides crucial physical restraint to reliably anchor the Permalloy flaps within the membrane when actuated (see Fig. 4e). PDMS (DuPont Sylgard 184) is spin-coated to achieve a $40-\mu \mathrm{m}$ membrane (Fig. 4c). The PDMS membrane is allowed to reflow to attain a planar top surface.

To release the membrane, the $30-\mu \mathrm{m}$ silicon layer needs to be removed. Despite the physical stability of PDMS, wet etchants can diffuse through the PDMS membrane at high temperatures (near $100^{\circ} \mathrm{C}$ ) and for extended periods ( $>1$ hour). Direct wafer immersion is therefore undesirable. Hence, we constructed a PDMS protective mold around the wafer for single-sided etching, leaving only the back window open (Fig. 4d). To avoid membrane damage due to strong PDMS-PDMS adhesion between membrane and mold (which causes membrane peel-off during mold removal), a thin, reusable spacer is used. A $1-\mathrm{mm}$ thick PDMS piece, 
entirely coated with $540-\AA$ thick $\mathrm{Cr}$ acts as this spacer (Fig. 4d). After the silicon window is etched through in $\mathrm{KOH}$, the PDMS mold is carefully removed to release the device (Fig. 4e).

(B) Diffuser Valve. The diffuser valves, pump chamber, microchannels (150- $\mu \mathrm{m}$ wide), and fluid inlet and outlet are defined onto a $175-\mu \mathrm{m}$ thick layer of SU-8 epoxy-based negative photoresist. PDMS molding produces Layer I in Figure 1.

\section{MEASUREMENTS AND RESULTS}

(A) Membrane Actuator. Preliminary experiments have been conducted to relate membrane displacements to the applied magnetic fields. Using a $\mathrm{NdFeB}$ permanent magnet (with a maximum surface magnetic field strength of $2.15 \times 10^{5}-\mathrm{A} / \mathrm{m}$ ) as the actuation source, deflections were measured at each comer of each Permalloy piece using an optical microscope.

Figure 9 shows membrane displacements recorded from successive measurements of the actuator at magnetic field strengths of $1.84 \times 10^{5}-\mathrm{A} / \mathrm{m}, 1.49 \times 10^{5}-\mathrm{A} / \mathrm{m}, 1.09 \times 10^{5}-\mathrm{A} / \mathrm{m}$, and $8.01 \times 10^{4}-\mathrm{A} / \mathrm{m}$, resulting in maximum displacements of $211.5-\mu \mathrm{m}$, $194.5-\mu \mathrm{m}, 157.5-\mu \mathrm{m}$, and $118.1-\mu \mathrm{m}$, respectively. ANSYS simulations produced a good match, with less than $6 \%$ error, between experimental results and theoretical expectations.

(B) Micropump. Flow rate versus frequency of applied field has been measured. Food dye placed over the fluid inlet is driven by an oscillating $1.99 \times 10^{5}-\mathrm{A} / \mathrm{m}$ external magnetic field. Flow measurements were recorded by observing fluid flow along the microchannels. A flow rate of $0.26-\mu \mathrm{l} / \mathrm{min}$ was measured for a $3.72-\mathrm{Hz}$ pumping frequency. The flow rate is a function of the frequency. Dynamic behavior is currently under study.

\section{CONCLUSIONS}

An actuator with a flexible, thin PDMS membrane with embedded magnetic pieces is made for the first time. This actuator is capable of achieving relatively large displacements, on the order of 100 to $200-\mu \mathrm{m}$. For this actuator, a novel fabrication technique for embedding ferromagnetic material within the thin PDMS membrane has been developed. In addition, design optimizations of the membrane actuator were completed using finite element analysis. The resultant magnetic membrane micropump is readily applicable to remotely-operated, implanted biomedical microfluidic systems. This is because it (i) is capable of telemetric operation without tether wires for power input, and (ii) does not require precise alignment between magnetic field and pump.

\section{REFERENCES}

1. M. Elwenspoek, et. al., "Towards integrated microliquid handling systems", J. Micromech. Microeng., 4, 227 (1994).

2. A. Olsson et. al., "Diffuser-element design investigation for valve-less pumps", Sensors \& Actuators, A57, 137 (1996).

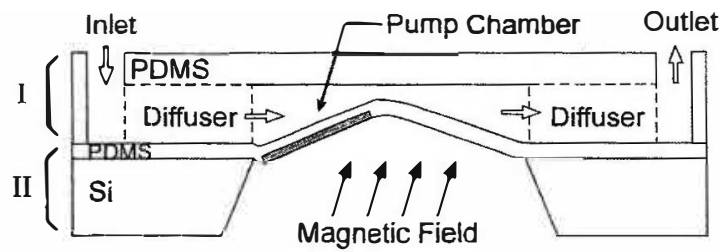

Figure 1. Cross section of assembled micropump.

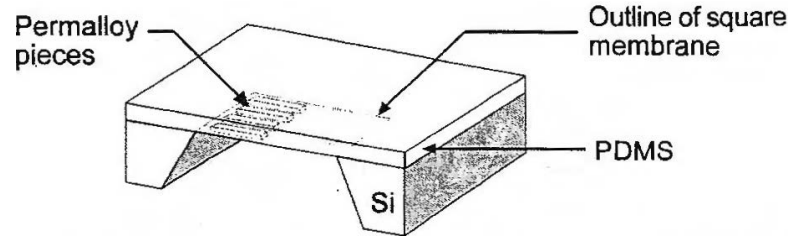

Figure 2. Schematic illustration of the membrane actuator (cut across its symmetry plane to illustrate the cross-section).

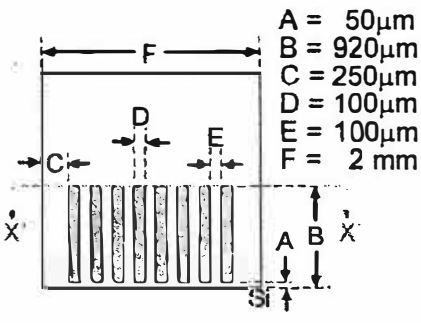

(a)

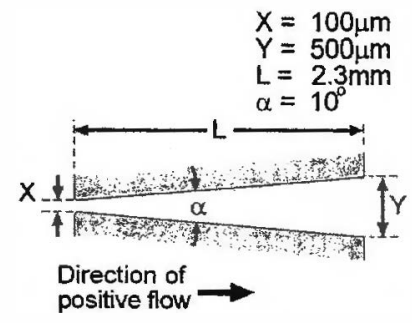

(b)

Figure 3.(a) Membrane actuator and (b) diffuser valve layout. $4000 \AA ̊$ oxidized $<100>$ silicon wafer with alignment marks

(a)

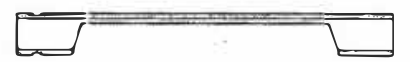

(b)

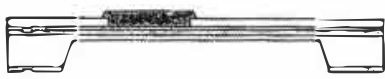

Etch Si in EDP, leaving a $30 \mu \mathrm{m}$ membrane

(c)

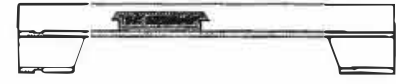

Deposit $\mathrm{Cr}-\mathrm{Cu}$ and over-
electroplate NiFe over PR electroplate NiFe over PR

(d)

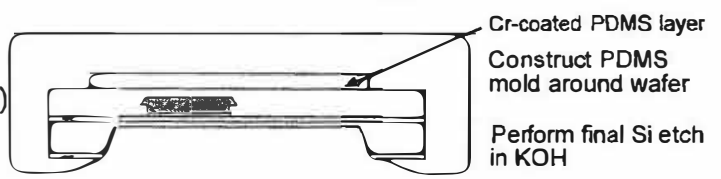

(e)

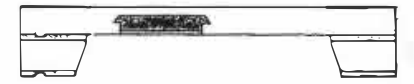

Remove PDMS mold and release membrane

Figure 4. Fabrication process for magnetic membrane actuator.

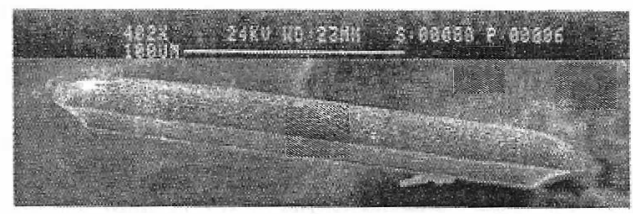

Figure 5. SEM micrograph showing shape at sides of overelectroplated Permalloy flap.

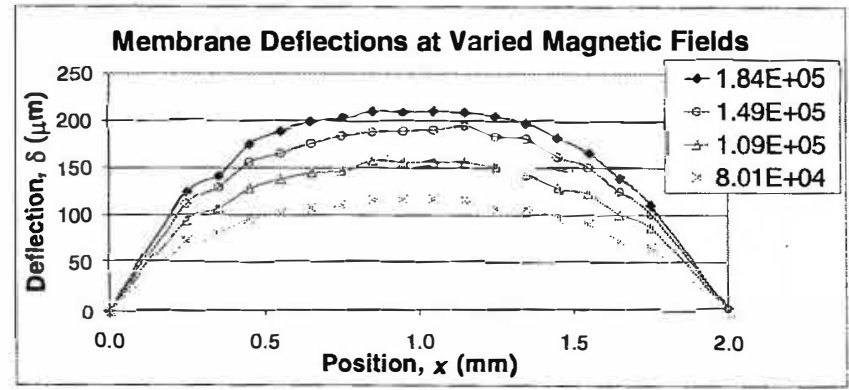

Figure 6. Plot shows vertical membrane displacements along the $\mathrm{X}^{\prime}-\mathrm{X}^{\prime}$ cross-section in Figure 3(a). 